\title{
“El Comportamiento del Tipo de Cambio en México y el Régimen de Libre Flotación: 1996-2001"
}

\author{
Santiago Bazdresch \\ santiago.bazdresch@yale.edu \\ Alejandro M. Werner ${ }^{*}$ \\ awerner@banxico.org.mx
}

Agosto de 2002

Documento de Investigación No. 2002-09

Dirección General de Investigación Económica

BANCO DE MÉXICO

\footnotetext{
Las opiniones presentadas aquí son las de los autores, y no necesariamente reflejan las del Banco de México. Agradecemos a Daniel Garcés sus comentarios y sus programas.
} 


\title{
"El Comportamiento del Tipo de Cambio en México y el Régimen de Libre Flotación: 1996-2001" \\ Santiago Bazdresch \\ Alejandro M. Werner
}

Agosto de 2002

Documento de Investigación No. 2002-09

Dirección General de Investigación Económica

BANCO DE MÉXICO

\begin{abstract}
$\underline{\text { Resumen }}$
El comportamiento del peso mexicano, desde la adopción del régimen de flotación, ha estado caracterizado por largos periodos de tranquilidad que repentinamente dan lugar a episodios de gran volatilidad. Para explicar este patrón de comportamiento, en el presente trabajo se desarrolla un modelo monetario de determinación del tipo de cambio, enriquecido con una prima de riesgo, de manera similar a la propuesta en el trabajo de Flood y Marion (1998). En el modelo, los cambios en las expectativas del mercado sobre el riesgo cambiario dan origen a equilibrios múltiples. En este modelo de determinación del tipo de cambio existen dos equilibrios, uno con pequeñas depreciaciones y poca volatilidad en el mercado cambiario y otro donde se presentan grandes depreciaciones y una alta volatilidad. La segunda parte del trabajo estima una regresión con cambio de régimen para explicar los movimientos diarios en el valor del peso respecto al dólar. Se encuentra evidencia significativa sobre la existencia de dos regímenes claramente identificables. Uno de ellos sin tendencia y con poca volatilidad y el otro con depreciaciones positivas y alta volatilidad. Finalmente, se utiliza el proceso estimado para explicar el sesgo implícito en los mercados de forwards del peso.
\end{abstract}




\section{Introducción}

Las crisis cambiarias y financieras experimentadas por el Sistema Monetario Europeo en 1992, por México en 1994-1995 y la reciente ola de crisis en los mercados emergentes en 1997-2001, han vuelto a encender el debate a cerca de los regímenes de tipo de cambio viables para economías pequeñas y abiertas $\mathrm{y}$, particularmente, para los mercados emergentes. En los países en cuestión, el desarrollo particular de cada crisis ha sido distinto. Sin embargo, tuvieron por lo menos un elemento común: el compromiso gubernamental de mantener un régimen de tipo de cambio predeterminado.

En México la crisis cambiaria de 1994-1995 hizo que la elección de un tipo de cambio flexible fuese la única viable. Aun cuando en ese entonces la opinión más generalizada resultaba que la flotación no era un régimen adecuado para México en el largo plazo, el día de hoy, más de seis años después, existe un consenso amplio en torno a los beneficios de este régimen y a su adecuación a las condiciones de la economía mexicana. Más recientemente, Brasil --que desde 1988 había mantenido el control del tipo de cambio como parte de un programa de estabilización-- también flexibilizó su régimen cambiario ante los embates especulativos de enero de 1999.

Después de más de seis años con el régimen de flotación, la experiencia de México constituye una referencia interesante para otras economías emergentes, que estén considerando un movimiento hacia regímenes de tipo de cambio más flexibles.

La evolución del peso mexicano bajo un régimen de flexibilidad ha sido similar al de otras monedas en flotación (Werner (1997)). A grandes rasgos, la volatilidad del peso mexicano durante su flotación, una vez controlada la crisis financiera y macroeconómica de 1995, ha sido similar a la experimentada por otras monedas con un régimen cambiario de flotación. Desde 1996, la instrumentación de políticas fiscales y monetarias prudentes, los programas aplicados para reestructurar el sistema financiero y la acumulación de reservas internacionales por el Banco de México $^{1}$, reestablecieron la confianza en el manejo macroeconómico, y como resultado, se ha observado un comportamiento ordenado del peso.

1 Las reservas internacionales pasaron de 16 mil millones de dólares en enero de 1996 a 28 mil millones en enero de 1998 y a 38 mil millones en junio de 2001. 
Tabla 1

Volatilidad del Tipo de Cambio de Varios Países con Respecto al Dólar

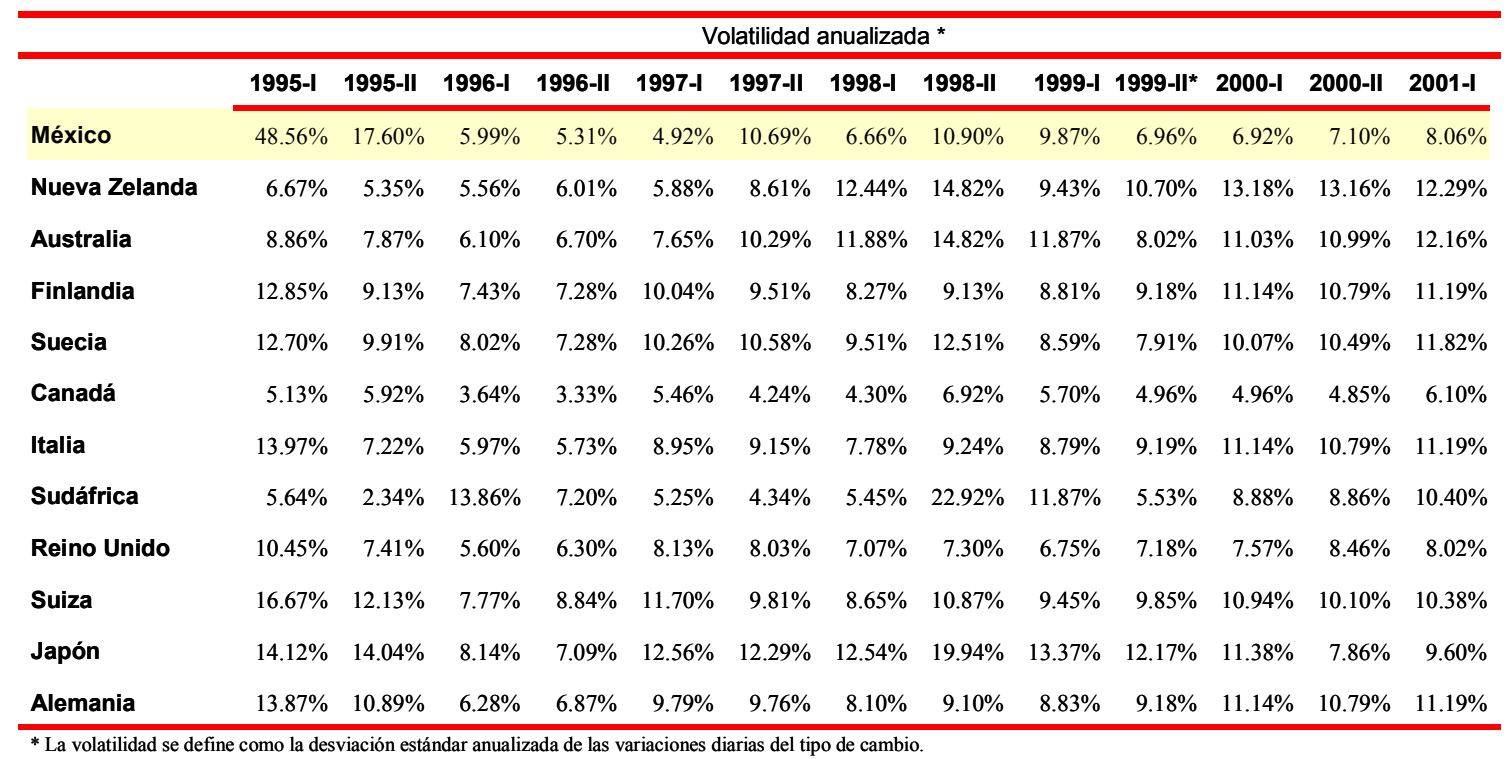

Sin embargo, la trayectoria del peso mexicano ha mostrado ciertos periodos de tranquilidad que luego han dado paso a periodos extremadamente volátiles. En la Gráfica 1 se muestra la volatilidad ${ }^{2}$ del tipo de cambio para México y Canadá, otra economía pequeña y abierta con un régimen cambiario de flotación. Es claro que el peso mexicano alterna, de tiempo en tiempo, entre episodios de calma y volatilidad. Estos últimos son mucho más marcados que para el caso canadiense.

2 Varianza de las innovaciones del tipo de cambio de los últimos 30 días. 


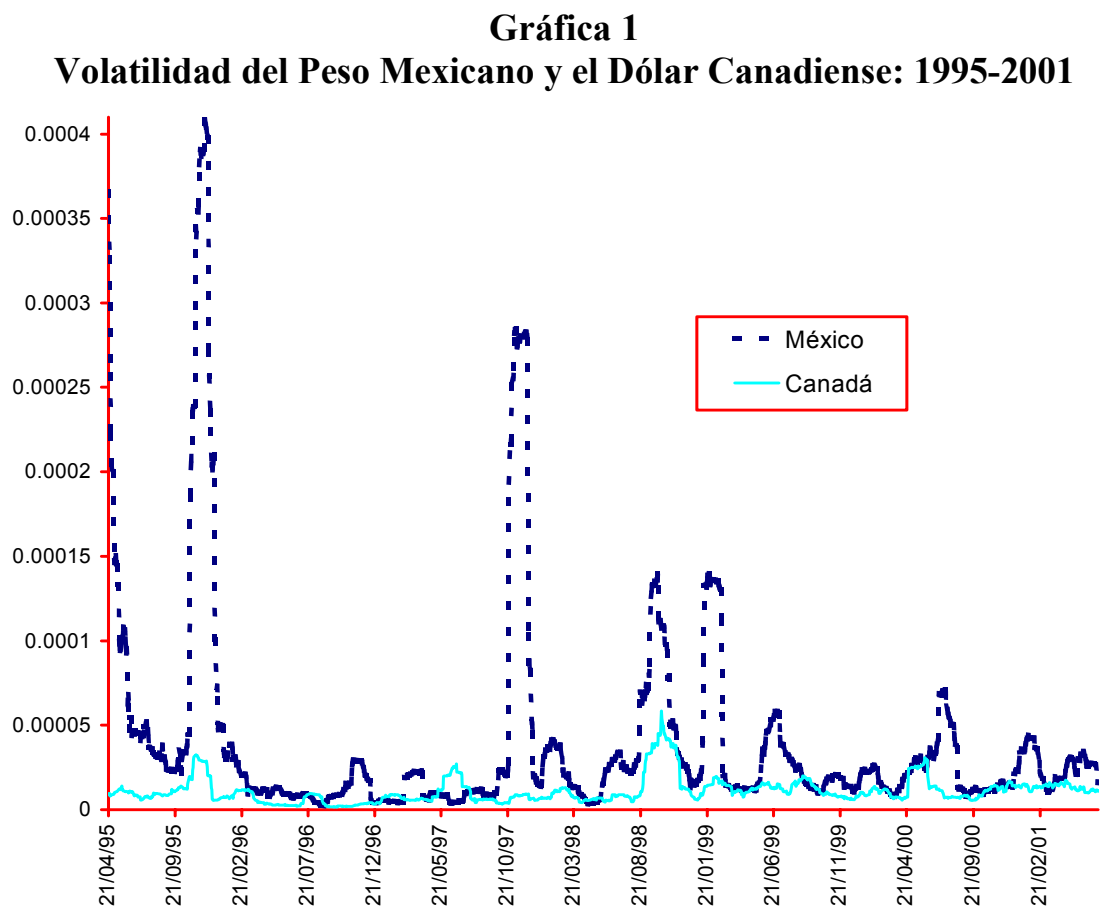

Para explicar este fenómeno, en la Sección 2 de esta nota se desarrolla un modelo monetario de determinación del tipo de cambio, aumentado con una prima de riesgo cambiario. Con esta prima de riesgo los cambios en las expectativas del mercado pueden verse autovalidadas y dar origen a equilibrios múltiples. El modelo tiene dos equilibrios, cada uno relacionado a una volatilidad cambiaria y depreciación distintas. Como era de esperarse, una mayor volatilidad va de la mano de una mayor depreciación. La existencia de estos dos equilibrios se constata a partir del modelo. El comportamiento del peso se describe simplemente diciendo que éste permanece la mayor parte del tiempo en el "buen equilibrio", con saltos periódicos debidos a presiones especulativas auto-realizadas, que están asociadas a movimientos transitorios hacia el "equilibrio malo".

En la Sección 3 se estima un modelo de cambio de régimen que presenta resultados que avalan las propiedades derivadas en el modelo teórico. Se encuentra evidencia estadísticamente significativa sobre la existencia de dos regímenes claramente identificables, uno con una tendencia nula o ligeramente negativa (apreciación) y con baja volatilidad; el otro, con depreciaciones considerables y alta volatilidad. En ambos, las perturbaciones están autocorrelacionadas negativamente; sin embargo, este resultado tiene poca significancia estadística. 
Finalmente, en la Sección 4 se utilizan las estimaciones del modelo de cambio de régimen para dar una explicación al "Problema del Peso" ${ }^{3}$. Este problema consiste en el sesgo encontrado en los mercados de forwards, los cuales llevan mucho tiempo pronosticando implícitamente devaluaciones del tipo de cambio peso/dólar superiores a las observadas. En el modelo, el Problema del Peso se explica como una probabilidad latente de pasar al régimen volátil y que se experimenten depreciaciones pronunciadas, aun cuando no se haya observado este régimen en un periodo largo.

\section{El Modelo de Determinación del Tipo de Cambio}

\section{II.1 Descripción del modelo}

En esta sección se utiliza un modelo monetario sencillo para subrayar una fuente potencial de multiplicidad en la determinación del tipo de cambio. La principal diferencia respecto a otros modelos simples de determinación del tipo de cambio ${ }^{4}$ es que se incluye su varianza, la cual se deriva de manera endógena y afecta la prima de riesgo cambiario y, por tanto, tiene un impacto sobre las tasas de interés. ${ }^{5}$

En este modelo la demanda de dinero es una función de la tasa de interés nominal, la que a su vez es determinada por la condición de paridad de tasas de interés. En consecuencia, la tasa de interés interna es igual a la tasa externa más las expectativas de depreciación de la moneda, más una prima de riesgo cambiario asociada a la incertidumbre de depreciación. Esto significa que los participantes en el mercado considerarán invertir en valores denominados en la moneda local dependiendo del rendimiento esperado, así como de la varianza o volatilidad de éste, exigiendo un mayor rendimiento esperado si hay una mayor volatilidad. Con el propósito de simplificar este análisis, se supone que se cumple la paridad del poder de compra, lo que permite utilizar al tipo de cambio nominal en lugar del nivel de precios. Las ecuaciones del modelo son las siguientes:

$$
\begin{aligned}
& m_{t}-s_{t}=-\alpha i_{t} \\
& i_{t}=i_{t}^{*}+E\left[s_{t+1}-s_{t}\right]+\theta_{t} b_{t}
\end{aligned}
$$

\footnotetext{
Conocido en la literatura como "Peso Problem".

El modelo es similar al desarrollado por Flood y Marion (1998), excepto que estos autores lo utilizan para explicar ataques especulativos a regímenes de tipo de cambio fijo.

5 Se refiere al lector a Werner (1996) y Babatz (1998) en cuanto a la evidencia empírica de una prima de riesgo cambiario para México.
} 
La ecuación (1) describe el mercado de dinero donde $m_{t}$ es el logaritmo de la oferta de base monetaria y $s_{t}$ es el logaritmo del tipo de cambio. La demanda de saldos reales depende negativamente de la tasa de interés interna, $i_{t}$, con una semielasticidad $\alpha$. La ecuación (2) es la condición de paridad de tasas de interés. La tasa de interés local es igual a la tasa de interés externa, $i_{t}{ }^{*}$, más la expectativa de devaluación, $E_{t}\left[s_{t+l^{-}} s_{t}\right]$, más una prima de riesgo, $\theta_{t} b_{t}$, que varía en el tiempo, donde el término $b_{t}$ representa el saldo de los bonos gubernamentales en poder del público y $\theta_{t}$ es una función del grado de aversión al riesgo y del nivel de riesgo cambiario que suponen aquellos inversionistas que toman posiciones en pesos.

Conviene describir a continuación los determinantes del coeficiente $\theta_{t}$ en el modelo. Este depende de la volatilidad del tipo de cambio y del grado de aversión al riesgo (z). Dado que la volatilidad del tipo de cambio $\left(\sigma_{s}^{2}\right)$ es endógena al modelo, el parámetro $\theta$ también estará determinado de manera endógena.

La evolución de la oferta de la base monetaria y de los bonos gubernamentales está determinada fuera del modelo; por lo que se supone que ambos son procesos estocásticos autorregresivos no correlacionados entre sí:

$$
\begin{aligned}
& m_{t}=\mu+m_{t-1}+\xi_{t} \\
& b_{t}=\eta+v b_{t-1}+\varepsilon_{t} \\
& \operatorname{Cov}\left(\xi_{i}, \varepsilon_{j}\right)=0 \quad \forall i, j
\end{aligned}
$$

\section{II.2 Solución del modelo}

Para resolver el modelo se utilizó el método de coeficientes indeterminados. Por tanto, se propone una solución con parámetros desconocidos y se sustituye esta solución en las ecuaciones originales para encontrar los valores apropiados de los parámetros. Puesto que el modelo es lineal, se propone una solución lineal para el tipo de cambio, como función de las variables de estado $\left(m_{t-1}, b_{t-1}\right)$ y de las perturbaciones estocásticas $\left(\xi_{t}, \varepsilon_{t}\right)$. La solución propuesta para el tipo de cambio tiene cinco parámetros de valor desconocido ( $\gamma_{0}$ hasta $\left.\gamma_{4}\right)$, esto es:

$s_{t}=\gamma_{0}+\gamma_{1} m_{t-1}+\gamma_{2} b_{t-1}+\gamma_{3} \varepsilon_{t}+\gamma_{4} \xi_{t}$

Una vez que se supone la forma funcional del tipo de cambio, es posible explicar el significado preciso del coeficiente $\theta$. Como se mencionó, este parámetro es igual a la 
varianza del tipo de cambio, multiplicada por un parámetro de aversión al riesgo. Su característica más importante es que, al igual que la varianza del tipo de cambio, está determinado endógenamente y dependerá de los parámetros de la solución propuesta para el tipo de cambio:

$$
\begin{aligned}
\sigma_{s_{t}}^{2}=\operatorname{Var}\left[s_{t}\right] & =E\left[\left(E\left[s_{t}\right]-s_{t}\right)^{2}\right] \\
& =E\left[\left(E\left[\gamma_{3} \xi_{t}+\gamma_{43} \varepsilon_{t}\right]-\gamma_{3} \xi_{t}+\gamma_{43} \varepsilon_{t}\right)^{2}\right] \\
& =\gamma_{3}^{2} \sigma_{\xi}^{2}+\gamma_{4}^{2} \sigma_{\varepsilon}^{2}
\end{aligned}
$$

por tanto:

$\theta=z \sigma_{s_{t}}^{2}=z\left[\gamma_{3}^{2} \sigma_{\xi}^{2}+\gamma_{4}^{2} \sigma_{\varepsilon}^{2}\right]$

La constante $z$ es el parámetro de aversión al riesgo, y $\sigma_{\varepsilon}^{2}$ y $\sigma_{\xi}^{2}$ son la varianza de las perturbaciones $\varepsilon$ y $\xi$, respectivamente.

Sustituyendo las ecuaciones (2), (3), (4) y (5) en (1) se obtienen las siguientes condiciones para que la solución propuesta sea un equilibrio de este modelo:

$\gamma_{0}=\alpha \eta \gamma_{2}+\alpha i^{*}+\mu+\alpha \gamma_{1} \mu+\alpha \eta \theta$

$\gamma_{1}=1$

$\gamma_{2}=\frac{\alpha v \theta}{1+\alpha-\alpha v}$

$\gamma_{3}=\frac{\alpha \gamma_{2}+\alpha \theta}{1+\alpha}$

$\gamma_{4}=1$

Sustituyendo (6) por $\theta$ en las ecuaciones (9) y (10) se llega al siguiente polinomio cuadrático para el valor de $\gamma_{3}$, que tiene la forma $A \gamma_{3}^{2}+B \gamma_{3}+C$ :

$$
\left[\alpha \sigma_{\varepsilon}^{2} z+\frac{\alpha^{2} v \sigma_{\varepsilon}^{2} z}{1+\alpha-\alpha v}\right] \gamma_{3}^{2}+[1+\alpha] \gamma_{3}+\left[\alpha \sigma_{\xi}^{2} z+\frac{\alpha^{2} v \sigma_{\xi}^{2} z}{1+\alpha-\alpha v}\right]=0
$$

De esta fórmula cuadrática se pueden encontrar dos soluciones para $\gamma_{3}$ que, de acuerdo con las ecuaciones (7) y (9), estarán asociadas a dos soluciones para $\gamma_{2}$ y $\gamma_{0}$, respectivamente. En la Gráfica 2 se muestra la función cuadrática de $\gamma_{3}$, con sus dos soluciones positivas, una mayor que la otra. Puesto que $\gamma_{3}$ es el parámetro asociado a la perturbación estocástica $\varepsilon$ se puede, de aquí en adelante, llamar a la solución que corresponde a un coeficiente $\gamma_{3}$ grande, 
la de varianza alta y a la otra solución, la de varianza baja. El resto de los parámetros tiene soluciones congruentes con la intuición económica y con el valor que tome $\gamma_{3}$. En la Gráfica 3 se ilustra que $\gamma_{0}$ y $\gamma_{2}$ son funciones crecientes de $\gamma_{3}$ mientras éste parámetro sea positivo, implicando que la solución de alta varianza de $\gamma_{3}$, estará asociada a depreciaciones mayores del tipo de cambio y la de baja varianza a depreciaciones pequeñas.

Los parámetros ${ }^{6}$ del modelo usados para construir estas funciones son: una elasticidad de la demanda de dinero de 0.3 , un parámetro de aversión al riesgo $(z)$ de 0.7 , un coeficiente de autocorrelación ( $v$ ) para los bonos de 0.9 , una varianza para $\varepsilon$ y $\xi$ de $\sigma_{\varepsilon}=2$ y $\sigma_{\xi}=0.5$ y una tasa de interés internacional $\left(i^{*}\right)=0.03$.

Gráfica 2

Función Cuadrática de $\gamma_{3}$.

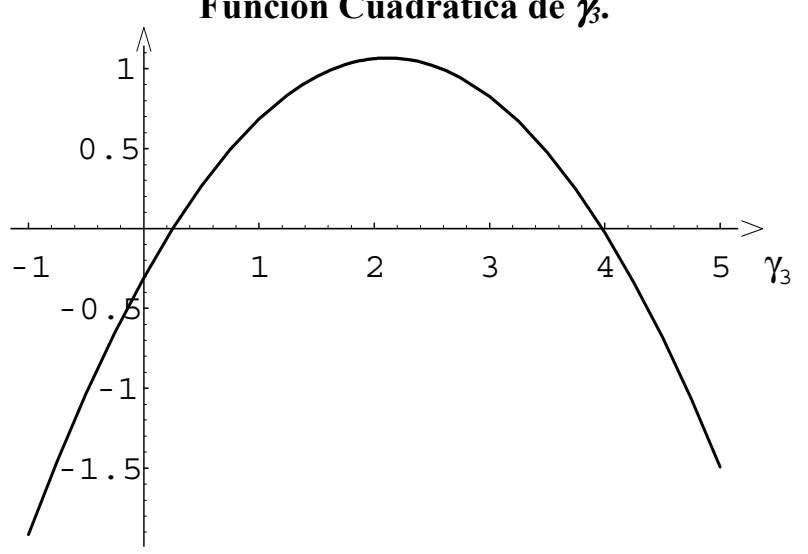

Gráfica 3

$\gamma_{0}$ y $\gamma_{2}$ como funciones de $\gamma_{3}$.

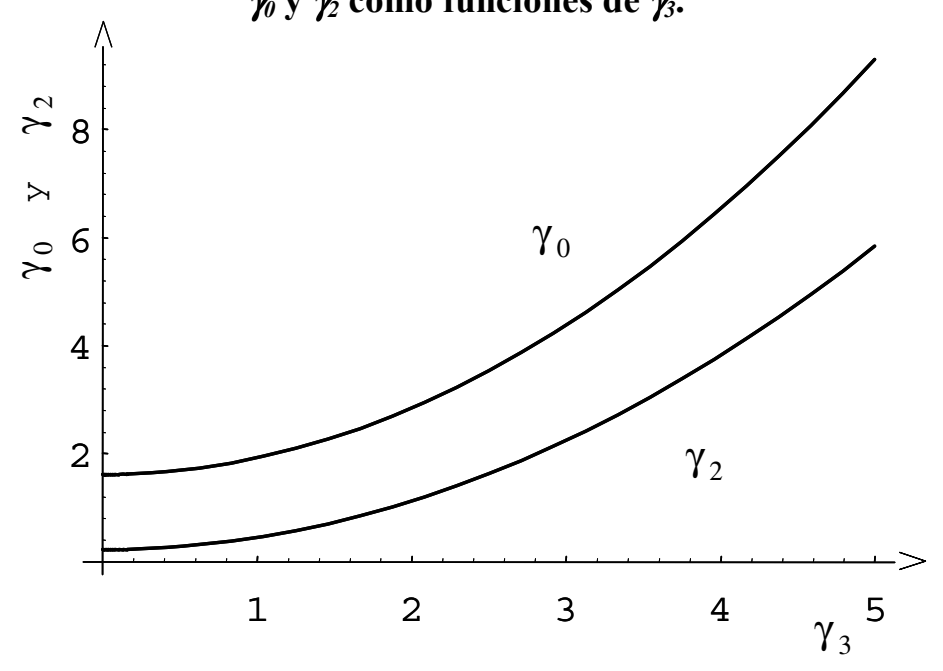

Para los valores de los coeficientes se utilizan valores similares a aquéllos usados por Flood y Marion (1998). 
Sin embargo, hasta este punto, no resulta intuitivo comprender cómo este modelo genera dos soluciones distintas para el proceso del tipo de cambio, por lo que vale la pena dar una explicación heurística. En este modelo se incluyó una prima de riesgo que se define como $\theta_{t} b_{t}$ donde $\theta_{t}=z \sigma_{s}^{2}$. La existencia de esta prima de riesgo es lo que genera la multiplicidad de soluciones. Si se supone, por un momento, que los agentes tienen una expectativa acerca de la variabilidad del tipo de cambio determinada exógenamente, entonces el parámetro $\theta$ se puede rescribir de la siguiente manera:

$\theta=z\left(\sigma_{s}^{2}\right)^{e}$

Si se utilizara la volatilidad esperada $\left(\sigma_{s}^{2}\right)^{e}$ en lugar de la volatilidad real (en la ecuación (12)) para calcular la percepción de riesgo de los mercados, se obtendría una tasa de interés interna determinada de la siguiente forma:

$i=i^{*}+\left(s_{t+1}^{e}-s_{t}\right)+z\left(\sigma_{s}^{2}\right)^{e} b_{t}$

Resolviendo nuevamente el modelo por el método de los coeficientes indeterminados, se obtiene lo siguiente:

$\gamma_{3}=\frac{\alpha\left(\sigma_{s}^{2}\right)^{e} z}{1+\alpha-\alpha \nu}$

$\sigma_{s}^{2}=\gamma_{3}^{2} \sigma_{\xi}^{2}+\sigma_{\varepsilon}^{2}$

El resultado indica que la volatilidad observada del tipo de cambio, $\sigma_{s}^{2}$, en cualquier momento, es una función de la volatilidad esperada por el mercado. En la Gráfica 4 se muestra que la función que relaciona la volatilidad esperada y la observada es cuadrática. La fuente de esta no linealidad es que la volatilidad esperada afecta al tipo de cambio linealmente, a través de la prima de riesgo y, consecuentemente, afecta a la volatilidad del tipo de cambio cuadráticamente. La función que relaciona a la volatilidad esperada con la observada tiene dos intersecciones con la línea de 45 grados. Estos son los puntos donde la volatilidad observada es igual a la esperada y, por tanto, constituyen las soluciones de expectativas racionales. 


\section{Gráfica 4}

Volatilidad Observada como Función de la Volatilidad Esperada

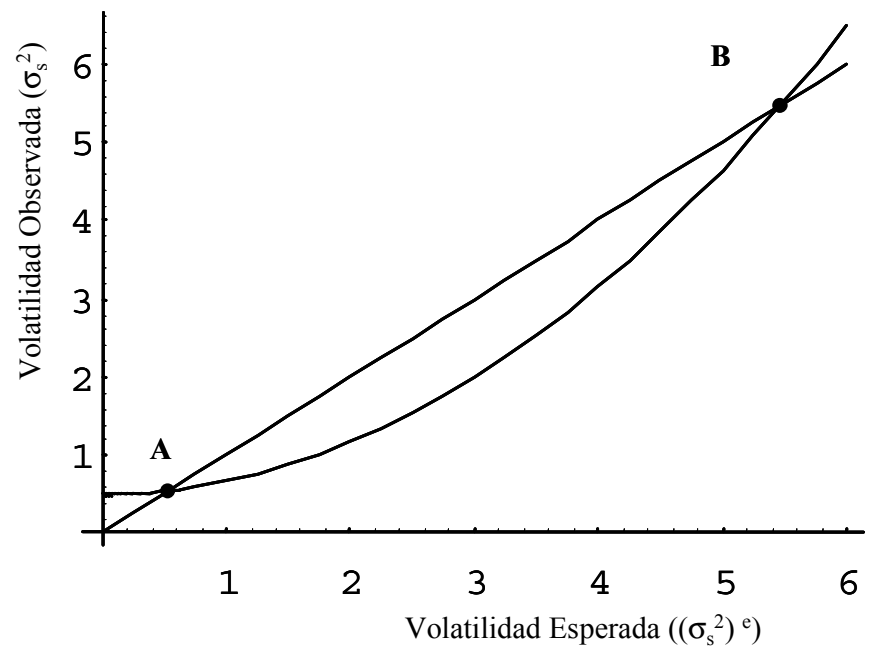

Este resultado permite interpretar el comportamiento del tipo de cambio como un proceso que alterna, ocasionalmente, entre una solución de varianza reducida y de depreciaciones pequeñas y otra, de volatilidad elevada y depreciaciones pronunciadas.

\section{Estimación del Modelo de Cambio de Régimen}

La solución para el tipo de cambio que se deriva en la Sección 2 se traduce en un proceso autorregresivo para las innovaciones del tipo de cambio, que toma la siguiente forma:

$\Delta s_{t}=(1-v) \gamma_{1} \delta+v \gamma_{2}(1-\eta)+v \Delta s_{t-1}+\varphi_{t-1}$

Este es un proceso autorregresivo para $\Delta s_{t}$, con una media que depende del estado de la naturaleza por medio de los coeficientes $\gamma_{1}$ y $\gamma_{2}$, y una varianza que depende del estado de la naturaleza a través de todos los coeficientes $\gamma_{1}$ hasta $\gamma_{4}{ }^{7}$

Una manera de estimar un proceso autorregresivo, con dos estados de la naturaleza distintos, es utilizando un modelo de cambio de régimen ${ }^{8}$. En estos modelos, cada elemento del conjunto de observaciones es explicado por una de varias ecuaciones o por regímenes distintos. En este caso, las innovaciones del tipo de cambio serán un proceso autorregresivo

\footnotetext{
$7 \mathrm{La}$ forma exacta del parámetro $\varphi$ en esta ecuación es $-v\left(\gamma_{1} \varepsilon_{t-1}+\gamma_{2} \xi_{t-1}+\gamma_{3}\left(\varepsilon_{t}-\varepsilon_{t-1}\right)+\gamma_{4}\left(\xi_{t}-\xi_{t-1}\right)\right)$ $+\left(\gamma_{1} \varepsilon_{t}+\gamma_{2} \xi_{t}+\gamma_{3}\left(\varepsilon_{t+1}-\varepsilon_{t}\right)+\gamma_{4}\left(\xi_{t+1}-\xi_{t}\right)\right)+\gamma_{2}(v-1) \xi_{t-1} ;$ a cada una de las dos soluciones de $\gamma_{3}$ en (11) corresponde un juego completo de parámetros $\Gamma=\left[\gamma_{0}, \gamma_{1}, \gamma_{2}, \gamma_{3}, \gamma_{4}\right]$.

8 Para una descripción detallada de este enfoque véase Hamilton (1994), c. 21.
} 
que alternará entre dos regímenes, donde todos los coeficientes del proceso pueden variar dependiendo del régimen en vigor. Las probabilidades de transición de un régimen a otro también se determinan endógenamente. El proceso de estimación asignará a cada observación una probabilidad $p$ de estar en el régimen volátil y de 1-p de estar en el régimen estable.

Se estimó el siguiente modelo para el periodo $1 / 1996-6 / 2001:^{9}$

$$
\begin{aligned}
\Delta s_{t} & =\mu_{i_{t}}+\rho_{i_{t}} \Delta s_{t-1}+\varepsilon_{t}^{\sigma_{t_{t}}} \quad i=0,1 \\
\Delta s_{t} & =\log \left(S_{t}\right)-\log \left(S_{t-1}\right) \\
\varepsilon_{t}^{\sigma_{i_{t}}} & \sim \text { i.i.d. } \mathrm{N}\left(0, \sigma_{i_{t}}\right)
\end{aligned}
$$

donde $i$ es el estado de la naturaleza, igual a 0 o 1 , correspondientes a los dos estados posibles.

Resultados importantes de la estimación de este modelo son la proporción de tiempo que el proceso permanece en cada estado, y la probabilidad de pasar de un estado a otro. Las probabilidades de transición de un estado a otro se expresan en la matriz $\mathrm{P}$, de la manera en que se presenta a continuación:

$P=\left[\begin{array}{ll}p_{11} & p_{21} \\ p_{12} & p_{22}\end{array}\right]$

donde $p_{i j}$ representa la probabilidad de ir al estado $j$ condicionado a estar en el estado $i$. El tiempo promedio que el proceso permanece en cada estado se calcula fácilmente a partir de esta matriz. En esta especificación queda implícito que se trata de una cadena de Markov, en tanto que las probabilidades de transitar a un régimen o a otro dependen exclusivamente del régimen al que pertenece la última observación, y no las anteriores.

Con esta especificación se espera que el tipo de cambio vaya de un estado a otro de acuerdo a las probabilidades estimadas, y que permanezca en cualquiera de estos estados durante varios periodos, de tal manera que represente adecuadamente el modelo de equilibrios múltiples descrito en la sección anterior.

Para estimar el modelo se utiliza el algoritmo propuesto por Hamilton (1990) el cual, mediante aproximaciones sucesivas, encuentra un punto óptimo $\Theta^{*}$, dentro del espacio

\footnotetext{
9 Se utiliza el precio diario del peso al cierre de operaciones.
} 
parametral. Este punto maximiza la función de verosimilitud (14), encontrando los parámetros que explican los datos de la mejor manera posible con el modelo propuesto. ${ }^{10}$

$L(\Theta)=\sum_{t=1}^{T} \log f\left(\Delta s_{t} \mid S_{t-1} ; \Theta\right)$

$f\left(\Delta s_{t} \mid S_{t-1} ; \Theta\right)=\pi_{t-1}\left(I_{t}=0\right) f\left(\Delta s_{t} \mid I_{t}=0, S_{t-1} ; \Theta\right)+\pi_{t-1}\left(I_{t}=1\right) f\left(\Delta s_{t} \mid I_{t}=1, S_{t-1} ; \Theta\right)$

donde $f(\mid)$ es la función de densidad condicional, $S_{t}$ es la serie del tipo de cambio hasta el punto $s_{t-l}$ y la sigla $I_{t}$ representa el régimen al que pertenece $s_{t}$.

Esta función de densidad condicional (15) es la de una variable que se distribuye normalmente, esto es, el error $\varepsilon_{\mathrm{t}}{ }^{\mathrm{\sigma i}}$ se distribuye de acuerdo a:

$$
\begin{aligned}
f\left(\Delta s_{t} \mid I_{t}=i, S_{t-1}, \theta\right)= & f\left(\Delta s_{t} \mid I_{t}=i, \Delta s_{t-1}, \theta\right) \\
& =\frac{1}{\sqrt{2 \pi \sigma^{2}}} \exp \left[\frac{-\left(\Delta s_{t}-\mu_{i}-\rho_{i} \Delta s_{t-1}\right)^{2}}{\sigma}\right]
\end{aligned}
$$

Los resultados de la estimación son los siguientes: ${ }^{11}$

\section{Cuadro 2}

Coeficientes del Modelo de Cambio de Régimen

\begin{tabular}{|cccc|c|}
\hline Estado & $\begin{array}{c}\text { Coeficiente } \\
\text { constante } \mu_{\mathrm{i}}\end{array}$ & $\begin{array}{c}\text { Coeficiente } \\
\text { autorregresivo } \rho_{\mathrm{i}}\end{array}$ & $\begin{array}{c}\text { Desviación } \\
\text { estándar } \sigma_{\mathrm{I}}\end{array}$ & \\
\hline 0 & -0.000203259 & -0.023629215 & 0.003675 & Estado de baja \\
& $(0.000116)$ & $(0.0221)$ & $(0.000921)$ & volatilidad \\
1 & 0.001761390 & -0.062716728 & 0.011097 & Estado de alta \\
& $(0.000785)$ & $(0.0726)$ & $(0.00394)$ & volatilidad \\
\hline
\end{tabular}

\section{Cuadro 3}

Resultados Probabilísticos del Modelo de Cambio de Régimen

\begin{tabular}{|c|cc|c|c|}
\hline \multicolumn{2}{|c|}{$\begin{array}{c}\text { Matriz de probabilidades de } \\
\text { transición } P\end{array}$} & $\begin{array}{c}\text { Probabilidades } \\
\text { ergódicas }\end{array}$ & $\begin{array}{c}\text { Duración esperada del } \\
\text { sistema en cada régimen }\end{array}$ \\
\hline Estado & 0 & 1 & & \\
\hline 0 & 0.9785 & 0.1027 & 0.827 & 47.62 \\
1 & 0.02144 & 0.8972 & 0.172 & 9.73 \\
\hline
\end{tabular}

${ }^{10}$ El algoritmo utiliza la rutina iterativa BFGS donde los valores iniciales son importantes para la convergencia. Sin embargo, el punto óptimo encontrado tiene propiedades de óptimo global.

11 Los coeficientes, excepto $\rho_{1}$, y $\rho_{2}$ son estadísticamente significativos al $5 \%$. 
La estimación de los coeficientes se presenta en el Cuadro 2, en donde se incluyen las medias $\left(\mu_{i}\right)$, desviaciones estándar $\left(\sigma_{i}\right)$ y coeficientes autorregresivos para los movimientos del tipo de cambio en cada unos de los regímenes. Hay una diferencia notable entre las varianzas de los dos regímenes. En el estado 1 hay una varianza que resulta más de tres veces superior a la del estado 0 . La media del estado de varianza reducida, $\mu_{0}$, es negativa y pequeña, implicando que este régimen va de la mano de pequeñas apreciaciones diarias del tipo de cambio (aproximadamente 0.02\%). La media del régimen de alta varianza es positiva y mucho más grande, implicando que el estado de alta varianza conlleva depreciaciones comparativamente grandes $(0.18 \%)$. Ambos coeficientes autorregresivos son negativos, implicando un cierto regreso a la media en los dos regímenes. Este resultado es sensible a la muestra. En particular, utilizando el periodo 1996-1999 se encontró un coeficiente autorregresivo negativo y significativo para el estado tranquilo y uno positivo para el estado volátil.

La matriz de probabilidades de transición es útil para relacionar intuitivamente el modelo con los datos. Esta indica que el estado de poca volatilidad es muy estable; de acuerdo al modelo, en promedio, la cadena de Markov permanece en este régimen 47.62 días a la vez. Dicho de otra manera, este es el plazo promedio de permanencia en este régimen antes de pasar al otro. El estado de alta volatilidad es considerablemente menos estable y, en promedio, la cadena de Markov permanece en este régimen 9.73 días cada vez que entra a él.

Finalmente, las estimaciones de las probabilidades ergódicas indican que el tipo de cambio está, por lo general, en el estado de baja varianza, pues permanece en éste $82.7 \%$ por ciento del tiempo. 


\section{Gráfica 5}

Tipo de Cambio y Probabilidad de Régimen de Alta Volatilidad

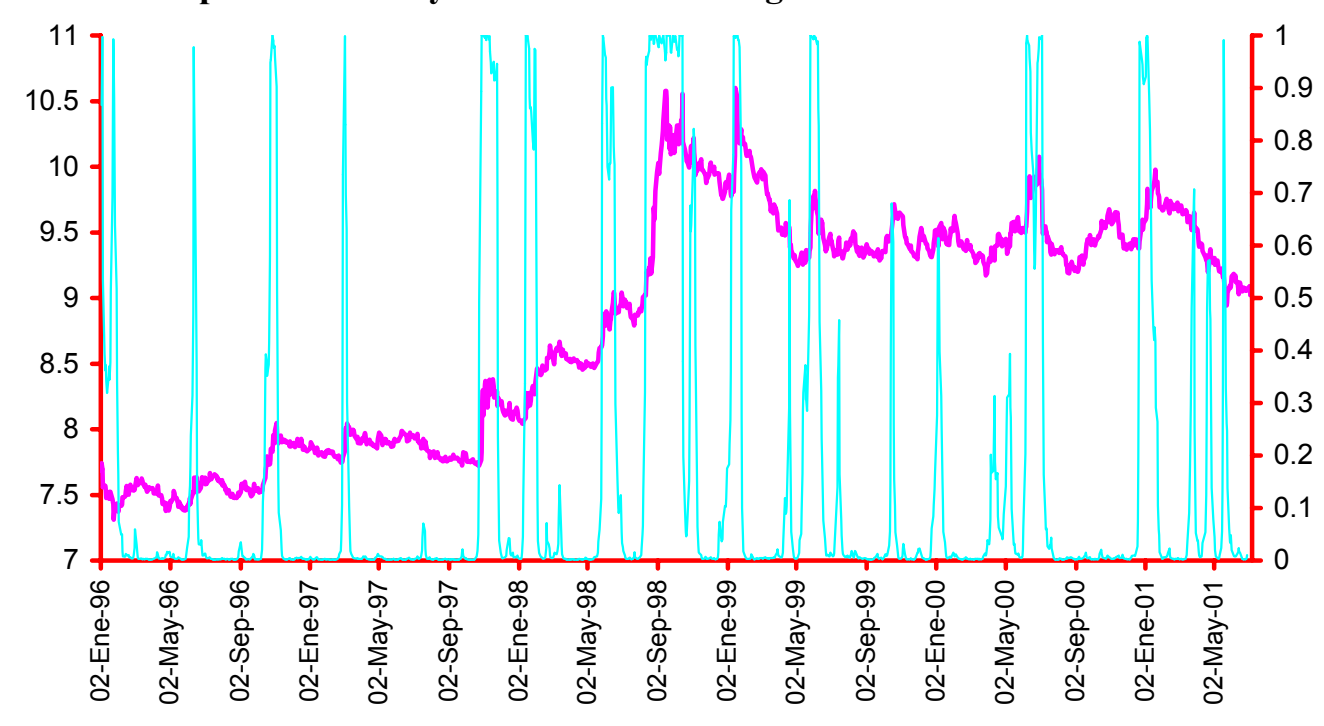

En la Gráfica 5 se presenta el precio de cierre del tipo de cambio junto con la probabilidad de que en dicha fecha el proceso del tipo de cambio haya pertenecido al régimen de alta volatilidad. Estas probabilidades son las llamadas probabilidades suavizadas ${ }^{12}$, calculadas tomando en cuenta la muestra completa de observaciones, y no sólo aquellas previas a ese punto en el tiempo. Estos estimados se ajustan adecuadamente a lo que se observa intuitivamente respecto al proceso seguido por el tipo de cambio.

\section{Explicación del Sesgo en el Precio a Futuro del Peso.}

\section{IV.1 Predicción}

En el Cuadro 4 se compara la precisión con la que se puede predecir el tipo de cambio del peso, utilizando diferentes métodos. Se comparan las predicciones obtenidas utilizando los valores forward del peso, un modelo autorregresivo estándar, y algunas variaciones del modelo de cambio de régimen presentado anteriormente. Los valores forward del peso son los contratos para intercambiar pesos por dólares en el futuro y representan en alguna medida las expectativas del mercado sobre el tipo de cambio en el futuro. Se espera que el modelo de cambio de régimen explique los movimientos del tipo de cambio mejor que el modelo autorregresivo típico, y que explique en alguna medida el sesgo observado en los precios a futuro del peso. 
En primer lugar, se estima el modelo de cambio de régimen para el periodo de enero de 1996 a diciembre de 1998, así como un modelo autorregresivo estándar para el mismo lapso. Dentro del esquema del modelo de cambio de régimen se utilizan como herramientas de predicción el modelo completo y el modelo condicional a permanecer en el estado tranquilo. El error cuadrático medio de las predicciones se calcula para cada especificación, para el periodo de enero de 1999 a junio de 2001. Dado el lapso considerado, estas pruebas indican la capacidad de predicción fuera de la muestra en estos modelos.

\section{Cuadro 4}

Comparación de Poder de Predicción con Distintos Modelos

\begin{tabular}{|c|cccc|}
\hline & $\begin{array}{c}\text { Mercado } \\
\text { (forwards) }\end{array}$ & $\begin{array}{c}\text { Modelo de cambio } \\
\text { De régimen: } \\
01 / 1996-12 / 1998\end{array}$ & $\begin{array}{c}\text { Modelo } \\
\text { autorregresivo }\end{array}$ & $\begin{array}{c}\text { Condicional a } \\
\text { estado tranquilo }\end{array}$ \\
\hline $\begin{array}{c}\text { Error cuadrático } \\
\text { medio }\end{array}$ & $5.43 \mathrm{e}-06$ & $1.55 \mathrm{e}-05$ & $1.40 \mathrm{e}-05$ & $5.44 \mathrm{e}-06$ \\
\hline
\end{tabular}

Del error cuadrático medio presentado en el Cuadro 4, es posible observar que los valores forward del peso mexicano producen ligeramente mejores predicciones que el modelo de cambio de régimen. Sin embargo, el modelo de cambio de régimen, condicional a quedarse en el régimen de poca volatilidad, produce predicciones casi tan buenas como las de los futuros del peso. Esto refleja el resultado de la alta probabilidad de estar en el estado tranquilo, y del proceso que ha seguido el tipo de cambio después de 1998.

\section{IV.2 El Problema del Peso}

Desde que México dejó flotar su moneda en diciembre de 1994, parece que los mercados han sobreestimado persistentemente el valor futuro del peso. Este tipo de comportamiento en el mercado de futuros y forwards es conocido como el "Peso-Problem" (Krasker (1980)). En esta sección se muestra que las expectativas del mercado pueden ser comprendidas si se construyen utilizando un modelo de cambio de régimen.

En el Cuadro 5 se presentan las devaluaciones diarias promedio que predicen los modelos anteriores así como sus errores de predicción. Se llama "predicción promedio" al promedio de las depreciaciones diarias que se extraen de las predicciones de cada modelo durante 1999, y "sesgo promedio" al error diario promedio en las predicciones de cada modelo respecto de la depreciación observada en el tipo de cambio.

\footnotetext{
12 Ver Hamilton (1994) y Kim (1994) para una explicación más detallada.
} 


\section{Cuadro 5}

Sesgo de Predicción

\begin{tabular}{|c|cccc|}
\hline & $\begin{array}{c}\text { Mercado } \\
\text { (forwards) }\end{array}$ & $\begin{array}{c}\text { Modelo de cambio } \\
\text { de régimen } \\
01 / 199612 / 1998\end{array}$ & $\begin{array}{c}\text { Modelo } \\
\text { autorregresivo }\end{array}$ & $\begin{array}{c}\text { Condicional a } \\
\text { estado tranquilo }\end{array}$ \\
\hline $\begin{array}{c}\text { Sesgo promedio de } \\
\text { predicción } \\
01 / 99-12 / 99\end{array}$ & 3.13 e-04 & 1.41 e-04 & 2.95 e-03 & $\mathbf{8 . 4 9}$ e-06 \\
\hline
\end{tabular}

El promedio de los errores de predicción del mercado durante el periodo de estudio es distinto de cero. Esto sucede incluso con las predicciones del modelo de cambio de régimen, el cual también tiene un error positivo en promedio. En promedio, este modelo explica $45.05 \%$ del error medio positivo de las expectativas del mercado. El modelo autorregresivo también tiene un error positivo en promedio, pero éste es mucho más grande que las expectativas de mercado con base en los contratos de futuros. El modelo de cambio de régimen, condicional a permanecer en el estado tranquilo, tuvo un sesgo promedio notablemente pequeño, lo cual puede atribuirse, a las condiciones inusualmente tranquilas de los mercados del peso después de 1998. Sin embargo, es el modelo completo el que tiene un sesgo más cercano al observado en el mercado. Se concluye que es la probabilidad de pasar al régimen de alta volatilidad lo que explica una parte importante del sesgo mostrado por los forwards del peso.

\section{Conclusiones}

En este trabajo se desarrolló un modelo muy simple para explicar algunos de los hechos estilizados del comportamiento del peso mexicano, desde la adopción del régimen de tipo de cambio flexible en diciembre de 1994. Siguiendo el trabajo realizado por Flood y Marion (1998) se derivó un modelo monetario de determinación del tipo de cambio en el cual se incluye una prima de riesgo. En este marco, las expectativas cambiantes del mercado acerca de la volatilidad del tipo de cambio pueden volverse auto-realizables y dar como resultado equilibrios múltiples, uno con poca volatilidad y pequeñas depreciaciones y otro con grandes depreciaciones y alta volatilidad. Además, se estimó un modelo de cambio de régimen para el tipo de cambio, el cual presenta evidencia clara de la existencia de dos regímenes con las propiedades anteriores. Finalmente, se mostró que este modelo explica más de $40 \%$ del sesgo presente en los forwards del peso mexicano. 


\section{Bibliografía}

Babatz, Guillermo (1998), "Exchange Rate Risk and the Debt Portfolio Composition of Mexican Firms", Capítulo 3, Tesis Doctoral, Universidad de Harvard.

Flood, Robert P. and Marion, Nancy P. (1998), "Self fulfilling risk predictions: an application to speculative attacks", Documento de trabajo del FMI, No. 98124.

Hamilton, James D. (1994), “Time Series Analisys”, Princeton University Press, Princeton.

Kaminsky, Graciela (1993), "Is There a Peso Problem? Evidence From the Dollar/Pound Exchange Rate, 1976-1987”, American Economic Review.

Kim,Chang-Jin (1994), "Dynamic Linear Models with Markov-switching", Journal of Econometrics, 60, 1-22, 1994.

Krasker, William (1980), “The 'Peso Problem' in Testing the Efficiency of Forward Exchange Markets”, Journal of Monetary Economics, 6, Abril, pp. 269-276.

Werner, Alejandro M. (1997), "Un estudio estadístico sobre el comportamiento de la cotización del peso mexicano frente al dólar y de su volatilidad", Banco de México, Documento de investigación 9701.

Werner, Alejandro M. (1996), “Mexico's Currency Risk Premia in 1992-1994: A Closer Look at the Interest Rate Differentials", Documento de trabajo del FMI, No. 96041. 